\title{
BoS3
}

\section{SAGEEP3 Multi-scale Monitoring of Ecohydrological Processes using Electrical Resistivity Tomography}

\author{
R.L. van Dam* (Michigan State University), D. Hyndman (Michigan State \\ University), A. Kendall (Michigan State University), K. Diker (Michigan \\ State University), B. Christoffersen (University of Arizona) \& S. Saleska \\ (University of Arizona)
}

\section{SUMMARY}

Hydrogeophysics is a growing discipline that holds significant promise to help elucidate details of dynamic processes in the near surface, built on the ability of geophysical methods to measure properties from which hydrological and geochemical variables can be derived. For example, bulk electrical conductivity is governed by, amongst others, interstitial water content, fluid salinity, and temperature, and can be measured using a range of geophysical methods. In many cases, electrical resistivity tomography (ERT) is well suited to characterize these properties in multiple dimensions and to monitor dynamic processes, such as water infiltration and solute transport.

In recent years, ERT has been used increasingly for ecosystem research in a wide range of settings; in particular to characterize vegetation-driven changes in root-zone and near-surface water dynamics. This increased popularity is due to operational factors (e.g., improved equipment, low site impact), data considerations (e.g., excellent repeatability), and the fact that ERT operates at scales significantly larger than traditional point sensors. Current limitations to a more widespread use of the approach include the high equipment costs, and the need for site-specific petrophysical relationships between properties of interest. In this presentation we will discuss recent equipment advances and theoretical and methodological aspects involved in the accurate estimation of soil moisture from ERT results. Examples will be presented from two studies in a temperate climate (Michigan, USA) and one from a humid tropical location (Tapajos, Brazil). 
Hydrogeophysics is a growing discipline that holds significant promise to help elucidate details of dynamic processes in the near surface, built on the ability of geophysical methods to measure properties from which hydrological and geochemical variables can be derived. For example, bulk electrical conductivity is governed by, amongst others, interstitial water content, fluid salinity, and temperature, and can be measured using a range of geophysical methods. In many cases, electrical resistivity tomography (ERT) is well suited to characterize these properties in multiple dimensions and to monitor dynamic processes, such as water infiltration and solute transport.

In recent years, ERT has been used increasingly for ecosystem research in a wide range of settings; in particular to characterize vegetation-driven changes in root-zone and near-surface water dynamics. This increased popularity is due to operational factors (e.g., improved equipment, low site impact), data considerations (e.g., excellent repeatability), and the fact that ERT operates at scales significantly larger than traditional point sensors. Current limitations to a more widespread use of the approach include the high equipment costs, and the need for site-specific petrophysical relationships between properties of interest. In this presentation we will discuss recent equipment advances and theoretical and methodological aspects involved in the accurate estimation of soil moisture from ERT results. Examples will be presented from two studies in a temperate climate (Michigan, USA) and one from a humid tropical location (Tapajos, Brazil). 